\title{
DIPOLE COLLAPSE IN ROTATING STRATIFIED DYNAMOS
}

\author{
L. Petitdemange ${ }^{1}$ and R. Raynaud ${ }^{2}$
}

\begin{abstract}
Numerical modelling of convection driven dynamos in the Boussinesq approximation revealed fundamental characteristics of the dynamo-generated magnetic fields, but the relevance of these results remains to be assessed for highly stratified systems, like gas planets and stars. The common approach is then to rely on the anelastic approximation to model the background density stratification. A conclusion from different anelastic studies is that dipolar solutions seem more difficult to obtain in presence of a substantial density contrast. We review some important results obtained by Raynaud et al. (2015), who investigated the influence of the density stratification on the stability of dipolar dynamos. This study indicates that the loss of the dipolar branch does not ensue from a specific modification of the dynamo mechanisms related to the background stratification, but could instead result from a bias as our observations naturally favour a certain domain in the parameter space characterized by moderate values of the Ekman number. In strongly stratified systems, the force balance may vary with depth, and a local increase of inertia close to the outer surface can explain the loss of the dipolar branch, while volume-averaged measures may underestimate the role of inertia on the field topology.
\end{abstract}

\section{Introduction}

The progress of Zeeman Doppler imaging continuously improve our picture of stellar magnetic fields, which are believed to originate from the action of self-excited dynamos induced by the turbulent motions that occur in stellar interior(See et al. 2016). At the same time, direct numerical simulations are still unable to resolve spatial and temporal scales over a realistic range, but one can use the anelastic approximation to filter out sound waves when assuming that the system stratification stays close to adiabatic. Just as in Boussinesq models (Christensen and

${ }^{1}$ LERMA, Observatoire de Paris, PSL Research University, CNRS, Sorbonne Universités, UPMC Univ. Paris 06, École normale supérieure, 75005 Paris, France

2 School of Astronomy, Institute for Research in Fundamental Sciences (IPM), 19395-5531, Tehran, Iran 
Aubert 2006; Schrinner et al. 2012; Yadav et al. 2013), magnetic fields obtained in anelastic simulations (Gastine et al. 2012; Duarte et al. 2013; Schrinner et al. 2014) fall into two categories: dipolar dynamos, dominated by a large-scale axial dipole component, and multipolar dynamos, characterized by a more complex field topology with higher spatial and temporal variability. However, these studies also pointed out that dipolar solutions seem more difficult to obtain as the density stratification is increased (Gastine et al. 2012; Jones 2014). In Raynaud et al. (2015), we investigated the evolution of the stability domain of dipolar dynamos when increasing the density stratification through a systematic parameter study including more than 200 three-dimensional, self-consistent dynamo models.

\section{Results}

\subsection{Bistability}

Figure 1 shows the distribution of dipolar and multipolar dynamos in the parameter space $\left(R a / R a_{\mathrm{c}}, P m\right)$, together with the corresponding dipolarities, for increasing density stratifications. One can see several examples of bistable pairs that are related to the use of stress-free boundary conditions that allows for the growth of stronger zonal winds (Sasaki et al. 2011; Schrinner et al. 2012; Gastine et al. 2012). For $N_{\varrho}=0.5$, the regime diagram in Figure 1a does not qualitatively differ from what we observe in the Boussinesq regime. As found by Raynaud et al. (2014), the multipolar branch undergoes a supercritical bifurcation as $R a$ is increased, whereas the dipolar one still loses its stability in favour of the multipolar branch at low Rayleigh and magnetic Prandtl numbers. When increasing the density contrast $N_{\varrho}$ to 1.5 , we see in Figure 1c that the overlap between the two branches shrinks. At $N_{\varrho}=2$, we do not observe a bistable case. More generally, for all models of our sample with a density stratification $N_{\varrho} \geq 2$, the saturated field of the dynamo is not anymore sensitive to the amplitude of the initial magnetic field. To understand this evolution from Boussinesq models to anelastic models with moderate stratification, it is worth stressing that the transition process from the multipolar to the dipolar branch triggered by the increase of $P m$ still applies to our sample of models, owing to the essential role played by differential rotation ( $\Omega$-effect) for multipolar dynamos (Schrinner et al. 2012).

\subsection{Dipole onset}

The density stratification strongly impacts on the stability domain of the dipolar branch. For moderate values of $N_{\varrho}$ at a fixed $P m$, the critical value of $R a / R a_{\mathrm{c}}$ at which it is possible to sustain a dipolar dynamo rapidly falls off (up to a factor four if we consider the line $P m=1$ ). However, this tendency hardly persists once we reach $R a / R a_{\mathrm{c}} \sim 5$ for $N_{\varrho}=1.5$, and the further increase of $N_{\varrho}$ mainly affects the critical magnetic Prandtl number $P m_{\mathrm{c}}$ below which it is not possible to sustain a dipolar dynamo. In our sample of models, the increase of $P m_{\mathrm{c}}$ becomes effective for $N_{\varrho} \geq 2$, but we already reported it as a general tendency in Schrinner et al. (2014). It is also directly responsible for the loss of the bistability which occurs for $N_{\varrho}=2$. (compare Figs. $1 \mathrm{c}$ and e). 


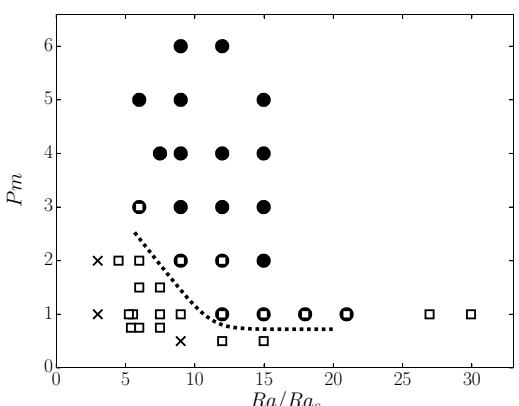

(a) $N_{\varrho}=0.5$

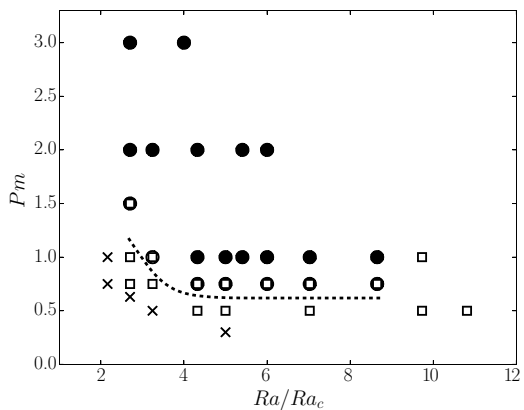

(c) $N_{\varrho}=1.5$

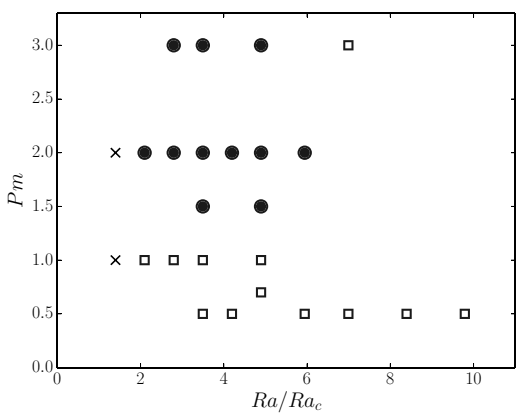

(e) $N_{\varrho}=2.0$

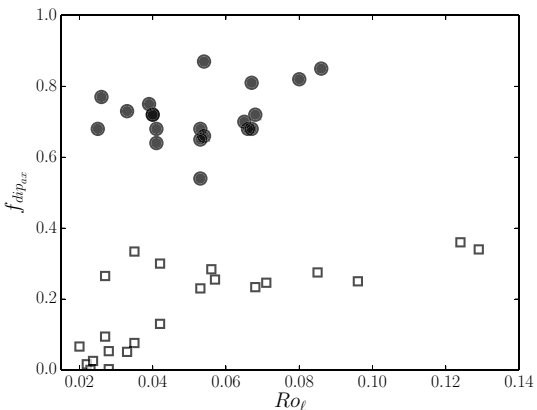

(b) $N_{\varrho}=0.5$

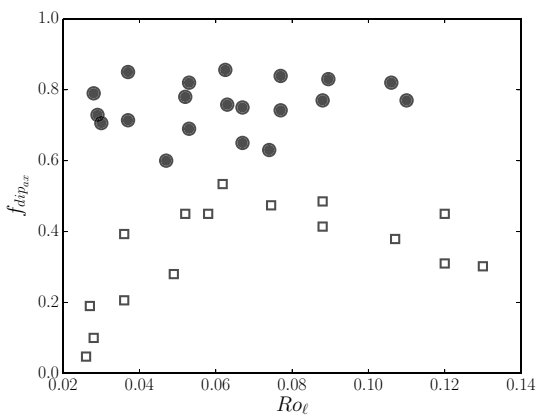

(d) $N_{\varrho}=1.5$

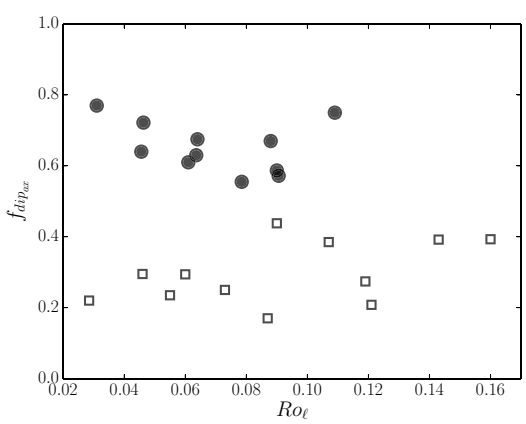

(f) $N_{\varrho}=2.0$

Fig. 1. Left: dipolar (black circles) and multipolar (white squares) dynamos as a function of $R a / R a_{\mathrm{c}}$ and $P m$, for $N_{\varrho}=0.5$ (a), $N_{\varrho}=1.5$ (c) and $N_{\varrho}=2.0$ (e). A cross indicates the absence of a self-sustained dynamo. Right: the relative axial dipole field strength $f_{\text {dip }_{\text {ax }}}$ versus the local Rossby number for $N_{\varrho}=0.5$ (b), $N_{\varrho}=1.5$ (d) and $N_{\varrho}=2.0$ (f). 


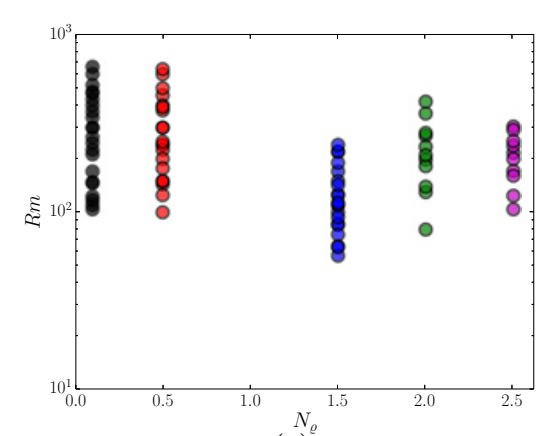

(a)

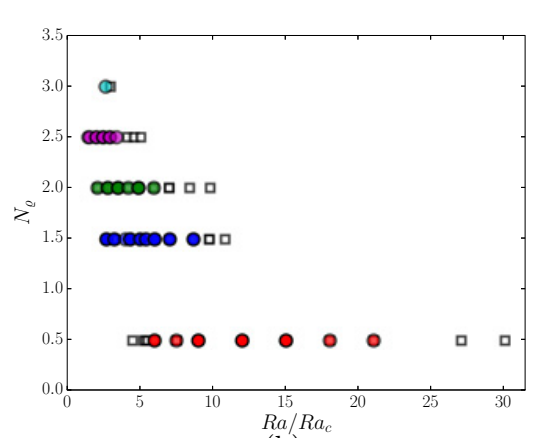

(b)

Fig. 2. (a) Magnetic Reynolds number as a function of $N_{\varrho}$ for our sample of dipolar dynamos. (b) Dipolar (circles) and multipolar (squares) dynamos in the parameter space $\left(R a / R a_{\mathrm{c}}, N_{\varrho}\right)$.

The fact that dipolar dynamos are found closer to the convection threshold as $N_{\varrho}$ increases can be more or less readily understood if one notices that, despite the increase of the density stratification, the critical magnetic Reynolds number $R m_{c}$ of the dipolar branch does not significantly varies, but stays in first approximation of order $10^{2}$, as shown in Figure 2a. If we take this as a necessary condition to obtain a dipolar solution, and given the fact that for a constant value of $R a / R a_{\mathrm{c}}$ the flow amplitude increases with $N_{\varrho}$ (Gastine et al. 2012), it explains why the dipolar branch is found closer to the onset of convection when the stratification increases. However, we see in the next subsection that, as $N_{\varrho}$ is further increased, not only does the dipolar branch occur closer to the onset of convection, but also higher $P m$ have to be considered to maintain a sufficiently high $R m$ while preventing the dipole collapse.

\subsection{Dipole collapse}

Another striking feature is that the range of Rayleigh numbers over which dipolar dynamos are sustained becomes smaller and smaller as $N_{\varrho}$ increases. This is illustrated in Figure $2 \mathrm{~b}$ that shows for different $N_{\varrho}$ the transition from the dipolar to the multipolar branch resulting from the increase of $R a$. In other words, at this moderate value of the Ekman number, dipolar dynamos are confined in a narrower and narrower window of Rayleigh numbers, which explains why dipolar solutions may seem more difficult to obtain at higher $N_{\varrho}$, despite comparable critical magnetic Reynolds numbers. As for the modification of the dynamo onset, this can be related to the fact that for a given value of $R a / R a_{\mathrm{c}}$, the Rossby number $R o$ increases with $N_{\varrho}$. The transition from a dipolar to a multipolar solution is related to the fact that inertia becomes significant in the force balance. We know from Christensen and Aubert (2006) that this transition can be measured by a local Rossby number $R o_{\ell}$ based on a characteristic length-scale of the flow. We find that the collapse of the dipole still occurs for $R o_{\ell} \sim 0.1$ when $N_{\varrho} \leq 2$, which is consistent 


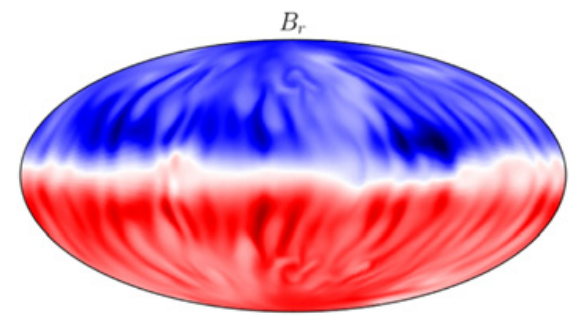

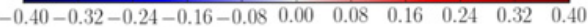

(a) $N_{\varrho}=1.5$

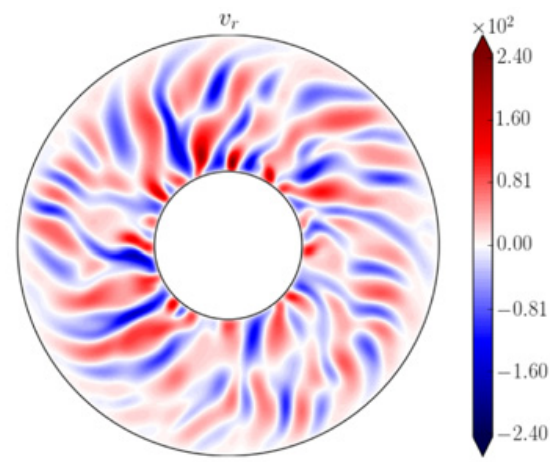

(b) $N_{\varrho}=1.5$

Fig. 3. Snapshot of $B_{r}\left(r=r_{o}\right)$ (a) and equatorial cut of $v_{r}$ (b) for a dipolar dynamo with $N_{\varrho}=1.5, P m=0.75, R a=4.625 \times 10^{6}=5 R a_{\mathrm{c}}$.

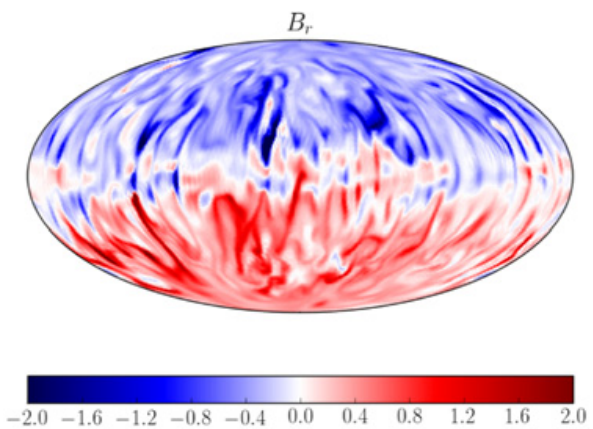

(a) $N_{\varrho}=2.5$

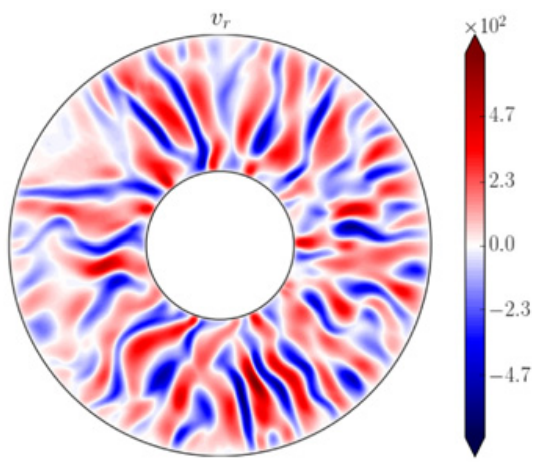

(b) $N_{\varrho}=2.5$

Fig. 4. Snapshot of $B_{r}\left(r=r_{o}\right)$ (a) and equatorial cut of $v_{r}$ (b) for a dipolar dynamo with $N_{\varrho}=2.5, P m=2, R a=7.40 \times 10^{6}=3.4 R a_{\mathrm{c}}$.

with the results in Gastine et al. (2012). This is illustrated by Figure 1b, d and $f$ which show the relative axial dipole field strength $f_{\text {dip }_{a x}}$ computed at the outer sphere, as a function of $R o_{\ell}$. Besides, we notice that the values of $\mathrm{f}_{\mathrm{dip}_{\mathrm{ax}}}$ tend to decrease with $N_{\varrho}$, which is also clear if we focus for instance on the dipolar branch for which $\mathrm{f}_{\text {dip }_{\mathrm{ax}}}<0.8$. As expected, this indicates that the small magnetic scales at the outer surface are favoured with the increase of the stratification. This is also clearly confirmed by the comparison of the radial magnetic fields at the outer surface of the model, as shown in (Figs. 3a and 4a). Finally, we also report the existence of multipolar dynamos whose dipolarity displays strong variations in time. This leads to averaged values of $\mathrm{f}_{\mathrm{dip}_{\mathrm{ax}}} \sim 0.5$, as one can notice in (Fig. $1 \mathrm{~d}$ ). 


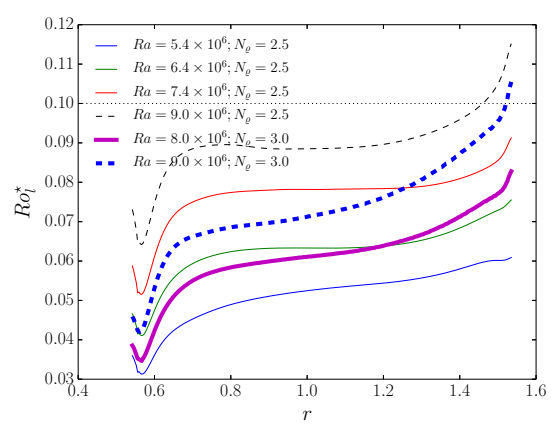

Fig. 5. The local Rossby number as a function of radius for dipolar (solid lines) and multipolar (dashed lines) dynamos at $\left(N_{\varrho}=2.5, P m=2\right)$ (thin lines) and $\left(N_{\varrho}=3\right.$, $P m=4)$ (thick lines).

These dynamos usually exhibit a relatively strong axial dipole component which undergoes reversals during which the value of $\mathrm{f}_{\mathrm{dip}_{\mathrm{ax}}}$ decreases drastically.

For $N_{\varrho}>2.0$, we found that the dipole collapse tends to occur at values of $R o_{\ell}$ lower than 0.1 . However, it is likely that a volume-averaged quantity becomes less relevant when applied to models with a substantial stratification. For instance, we see in Figures $3 \mathrm{~b}$ and $4 \mathrm{~b}$ that the smaller structures that develop at $N_{\varrho}=2.5$ are confined close to the outer boundary, whereas there are no significant differences in the radial flow at mid-depth. Thus, we also examined the radial dependence of the different components of the local Rossby number $R o_{l}^{\star}$, which is computed as the product of two terms: a convective Rossby number based on the velocity field $v_{c}$ from which the mean zonal flow has been subtracted and a characteristic lengthscale based on the mean harmonic degree of $v_{c}$. We find that the monotonicity of $R o_{l}^{\star}$ changes as $N_{\varrho}$ is increased. Indeed, for low stratifications, $R o_{l}^{\star(r)}$ mainly decreases with radius, whereas for $N_{\varrho} \geq 2.5$ it becomes an increasing function of $r$ that steepens slightly close to the outer surface. Figure 5 shows the evolution of $R o_{l}^{\star}(r)$ for increasing Rayleigh numbers up to the loss of the dipolar solution, at $N_{\varrho}=2.5$ and 3.0. When the transition to the multipolar branch is reached, we see that $R o_{\ell}^{\star}$ tends to increase faster close to the outer surface, while the volumeaveraged value can stay below the critical value of 0.1 . Thus, it seems that inertia still causes the collapse of the dipolar branch, despite the fact that the usual local Rossby number criterion is not appropriate to separate the two dynamo branches for significant density stratifications.

\section{Conclusion}

With this systematic parameter study, we are able to improve our understanding of the successive modifications that are exhibited by the stability domain of the dipolar branch when increasing the density stratification in anelastic dynamo models. In general, dipolar dynamos are found closer to the onset of convection. 
We also show that dipole-dominated solutions can be observed even at high density stratifications, provided high enough $P m$ are considered. Besides, this study also highlights why dipolar dynamos seem more difficult to find in anelastic simulations: we show that this general impression mainly results from the fact that the dipolar branch extends on a smaller and smaller range of Rayleigh numbers as $N_{\varrho}$ is increased. However, despite the relative shrinking of the stability domain, we found that the critical magnetic Reynolds number of the dipolar branch seems scarcely modified in the overall process. At the same time, the higher $N_{\varrho}$, the faster convection will develop as we depart from the onset. In consequence, the higher $N_{\varrho}$, the faster is reached the critical Rossby number above which inertia causes the collapse of the dipole. This explains why dipolar dynamos become clearly confined in a smaller region of the parameter space, but we must stress that, in terms of magnetic Reynolds number, the dynamo threshold does not significantly increase with the density stratification in the range of $N_{\varrho}$ we investigated. This suggests that the scarcity of dipolar solutions for substantial density stratifications would thus rather come from the restriction of the parameter space being currently explored (because of computational limitations), rather than an intrinsic modification of the dynamo mechanisms that would be caused by the density stratification.

\section{References}

Christensen, U.R. \& Aubert, J., 2006, Geophys. J. Int., 166, 97

Duarte, L.D.V., Gastine, T., \& Wicht, J., 2013, Phys. Earth Planet. Inter., 222, 22

Gastine, T., Duarte, L., \& Wicht, J., 2012, Astron. Astrophys., 546, A19

Jones, C., 2014, Icarus, 241, 148

Raynaud, R., Petitdemange, L., \& Dormy, E., 2014, Astron. Astrophys. 567, A107

Raynaud, R., Petitdemange, L., \& Dormy, E., 2015, MNRAS, 448, 2055

Sasaki, Y., Takehiro, S.-i., Kuramoto, K., \& Hayashi, Y.-Y., 2011, Phys. Earth Planet. Inter. 188, 203

Schrinner, M., Petitdemange, L., \& Dormy, E., 2012, Astrophys. J., 752, 121

Schrinner, M., Petitdemange, L., Raynaud, R., \& Dormy, E., 2014, Astrono. Astrophys., $564, \mathrm{~A} 78$

See, V., Jardine, M., Vidotto, A.A., Donati, J.-F., Boro Saikia, S., Bouvier, J., Fares, R., Folsom, C.P., Gregory, S.G., Hussain, G., Jeffers, S.V., Marsden, S.C., Morin, J., Moutou, C., do Nascimento, J.D., Petit, P., \& Waite, I.A., 2016, MNRAS, 462, 4442

Yadav, R.K., Gastine, T., Christensen, U.R., \& Duarte, L.D.V., 2013, Astrophys. J., $774(1), 6$ 